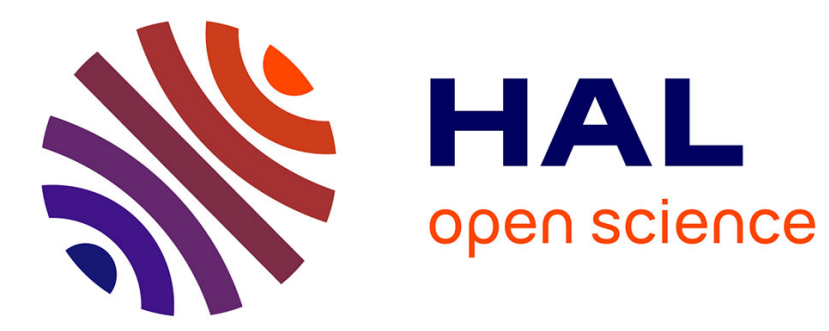

\title{
Random-field model for the elasticity tensor of anisotropic random media
}

Christian Soize

\section{To cite this version:}

Christian Soize. Random-field model for the elasticity tensor of anisotropic random media. Comptes Rendus Mécanique, 2004, 332 (12), pp.1007-1012. 10.1016/j.crme.2004.09.008 . hal-00686196

\section{HAL Id: hal-00686196 https://hal.science/hal-00686196}

Submitted on 8 Apr 2012

HAL is a multi-disciplinary open access archive for the deposit and dissemination of scientific research documents, whether they are published or not. The documents may come from teaching and research institutions in France or abroad, or from public or private research centers.
L'archive ouverte pluridisciplinaire HAL, est destinée au dépôt et à la diffusion de documents scientifiques de niveau recherche, publiés ou non, émanant des établissements d'enseignement et de recherche français ou étrangers, des laboratoires publics ou privés. 


\title{
Random-field model for the elasticity tensor of anisotropic random media
}

\author{
Christian Soize ${ }^{a}$ \\ ${ }^{a}$ Laboratoire de Mécanique, Université de Marne-la-Vallée, 5 Bd Descartes, 77454 Marne-la-Vallée, France
}

\begin{abstract}
This paper deals with the construction of a non-Gaussian positive definite matrix-valued random field whose mathematical properties allow the fourth-order elasticity tensor of random non homogeneous anisotropic three dimensional elastic media to be modelled. If the usual parametric probabilistic approach was used, then 21 mutually dependent random fields should be modelled and identified by using experimental data. Such an approach would be very difficult because the systems of the marginal probability distributions of these random fields have to be identified due to the fact that, for a boundary value problem, the displacement field of the random medium is a non-linear mapping of the random elasticity tensor. The theory presented in this paper allows such a probabilistic model of the fourth-order elasticity tensor field to be constructed and depends only of four scalar parameters: three spatial correlation lengths and one parameter allowing the level of the random fluctuations to be controlled. To cite this article: C. Soize, C. R. Mecanique (2004).
\end{abstract}

\section{Résumé}

Modèle de champ aléatoire pour le tenseur d'élasticité des milieux aléatoires anisotropes. On présente la construction d'un champ aléatoire à valeurs dans les matrices définies positives dont les propriétés mathématiques permettent de modéliser le tenseur d'élasticité du quatrième ordre des mileux élastiques anisotropes tridimensionnels aléatoires. Si l'approche probabiliste paramétrique usuelle était utilisée, alors il serait nécessaire de modéliser et d'identifier à l'aide de données expérimentales 21 champs aléatoires mutuellement dépendants. Une telle approche serait très difficile de part le fait que le système de lois marginales de ces champs aléatoires doit être identifié parce que, pour un problème aux limites, le champ de déplacement est une transformation non linéaire du tenseur d'élasticité. La théorie présentée dans ce papier permet de construire une modélisation probabiliste du champ de tenseur d'élasticité qui ne dépend que de quatre paramètres scalaires : trois échelles de corrélation spatiale et un paramètre permettant de contrôler le niveau des fluctuations aléatoires. Pour citer cet article : C. Soize, C. R. Mecanique (2004).

Key words: stochastic anisotropic elasticity; random medium; non-Gaussian random field

Mots-clés : Elasticité anisotrope stochastique; milieu aléatoire; champ aléatoire non gaussien

Email address: soize@univ-mlv.fr (Christian Soize).

Preprint submitted to Elsevier Science

April 8, 2012 


\section{Introduction}

An important problem is the construction of a stochastic representation for the fourth-order elasticity tensor of random non homogeneous anisotropic three-dimensional elastic media. Let us consider the following deterministic elliptic partial differential operator $\underline{A}$ on a bounded open domain $\Omega$ of $\mathbb{R}^{3}$, related to the three-dimensional linear elasticity for a non homogeneous anisotropic elastic material,

$$
\underline{A} \underline{\boldsymbol{u}}=-\sum_{i=1}^{3} \boldsymbol{e}^{i} \sum_{j=1}^{3} \frac{\partial}{\partial x_{j}}\left\{\sum_{k, h=1}^{3} \underline{c}_{i j k h}(\boldsymbol{x}) \varepsilon_{k h}(\underline{\boldsymbol{u}})\right\}
$$

in which $\boldsymbol{x}=\left(x_{1}, x_{2}, x_{3}\right) \in \Omega \subset \mathbb{R}^{3}$, where $\left\{\boldsymbol{e}^{1}, \boldsymbol{e}^{2}, \boldsymbol{e}^{3}\right\}$ are the three vectors of the canonical basis of $\mathbb{R}^{3}$ and where $\boldsymbol{x} \mapsto \underline{\boldsymbol{u}}(\boldsymbol{x})=\left(\underline{u}_{1}(\boldsymbol{x}), \underline{u}_{2}(\boldsymbol{x}), \underline{u}_{3}(\boldsymbol{x})\right)$ is a twice differentiable function from $\Omega$ into $\mathbb{R}^{3}$. The second-order strain tensor is such that $\varepsilon_{k h}(\underline{\boldsymbol{u}})=(1 / 2)\left(\partial \underline{u}_{k} / \partial x_{h}+\partial \underline{u}_{h} / \partial x_{k}\right)$. The fourth-order elasticity tensor $\underline{c}_{i j k h}(\boldsymbol{x})$ has to verify [1] the symmetry property $\underline{c}_{i j k h}(\boldsymbol{x})=\underline{c}_{j i k h}(\boldsymbol{x})=\underline{c}_{i j h k}(\boldsymbol{x})=\underline{c}_{k h i j}(\boldsymbol{x})$ and, for all symmetric second-order real tensors $\left\{z_{i j}\right\}_{i j}$, has to verify the positive-definiteness property, $\sum_{i, j, k, h=1}^{3} \underline{c}_{i j k h}(\boldsymbol{x}) z_{k h} z_{i j} \geq \underline{c}_{0} \sum_{i, j=1}^{3} z_{i j}^{2}$, in which $\underline{c}_{0}$ is a positive constant independent of $\boldsymbol{x}$. For a random medium, for all $\boldsymbol{x}$ fixed in $\Omega$, the tensor $\left\{\underline{c}_{i j k h}(\boldsymbol{x})\right\}_{i j k h}$ is replaced by a fourth-order tensor-valued random variable $\left\{C_{i j k h}(\boldsymbol{x})\right\}_{i j k h}$ whose mean value is $\left\{\underline{c}_{i j k h}(\boldsymbol{x})\right\}_{i j k h}$ and which has to verify the symmetry and the positive-definiteness properties in a probabilistic sense which has to be defined. Nevertheless, for the random case, the deterministic constant $\underline{c}_{0}$ (introduced above) cannot generally be justified from a probabilistic modelling point of view. Finally, $\boldsymbol{x} \mapsto\left\{C_{i j k h}(\boldsymbol{x})\right\}_{i j k h}$ is a fourth-order tensor-valued random field indexed by $\Omega$, constituted of 21 mutually dependent random fields and the stochastic partial differential operator $\boldsymbol{A}$ associated with the operator $\underline{A}$ written as

$$
\boldsymbol{A} \boldsymbol{U}=-\sum_{i}^{3} \boldsymbol{e}^{i} \sum_{j=1}^{3} \frac{\partial}{\partial x_{j}}\left\{\sum_{k, h=1}^{3} C_{i j k h}(\boldsymbol{x}) \varepsilon_{k h}(\boldsymbol{U})\right\}
$$

It should be noted that the probability distribution of this fourth-order tensor-valued random field (that is to say the system of the marginal probability distributions) is required because the unknown solution of the stochastic boundary value problem is a non-linear mapping of the random field $\boldsymbol{x} \mapsto\left\{C_{i j k h}(\boldsymbol{x})\right\}_{i j k h}$. If the usual parametric probabilistic approach is used, then the identification of this probability model by using experimental data seems to be difficult. This paper deals with a non-parametric construction of the random field $\boldsymbol{x} \mapsto\left\{C_{i j k h}(\boldsymbol{x})\right\}_{i j k h}$. For that, an ensemble of non-Gaussian positive-definite matrix-valued random fields is constructed and studied. With such a construction, the tensor-valued random field will depend only on 4 scalar parameters: three spatial correlation lengths and one parameter allowing the 
level of the random fluctuations to be controlled. With such a model, the inverse problem related to the experimental identification seems to be more feasible.

Let $\boldsymbol{x}=\left(x_{1}, \ldots, x_{n}\right)$ be a vector in the Euclidean space $\mathbb{R}^{n}$ equipped with the inner product $\langle\boldsymbol{x}, \boldsymbol{y}\rangle=$ $\sum_{j=1}^{n} x_{j} y_{j}$ and the associated norm $\|\boldsymbol{x}\|=<\boldsymbol{x}, \boldsymbol{x}>^{1 / 2}$. Let $\mathbb{M}_{n}(\mathbb{R})$ be the set of all the square $(n \times n)$ real matrices, $\mathbb{M}_{n}^{S}(\mathbb{R})$ the subset of the symmetric matrices and $\mathbb{M}_{n}^{+}(\mathbb{R})$ the subset of the symmetric positive-definite matrices. For a matrix $[A] \in \mathbb{M}_{n}(\mathbb{R})$, one introduces the usual following notation: $[A]^{T}$ the transpose, $\operatorname{tr}[A]=\sum_{j=1}^{n}[A]_{j j},\|A\|_{F}^{2}=\operatorname{tr}\left\{[A]^{T}[A]\right\}$ and $\|A\|=\sup _{\|\boldsymbol{x}\| \leq 1}\|[A] \boldsymbol{x}\|$. One has $\|A\| \leq$ $\|A\|_{F} \leq \sqrt{n}\|A\|$.

\section{Ensemble $\mathrm{SFG}^{+}$of normalized non-Gaussian positive-definite matrix-valued random fields}

\subsection{Random field $S$ as the germ of the ensemble $S F G^{+}$}

One introduces a random field $S$ as the germ of the normalized non-Gaussian positive-definite matrixvalued random fields belonging to $\mathrm{SFG}^{+}$which are defined as a non-linear mapping of independent copies of the germ $S$.

Definition. Let $d \geq 1$ be an integer. Let $\boldsymbol{x} \mapsto S(\boldsymbol{x})$ be a second-order centered homogeneous Gaussian random field, defined on the probability space $(\Theta, \mathcal{T}, P)$, indexed by $\mathbb{R}^{d}$, with values in $\mathbb{R}$. Let $L_{1}, \ldots, L_{d}$ be positive real numbers. The autocorrelation function $R_{S}(\boldsymbol{\eta})=E\{S(\boldsymbol{x}+\boldsymbol{\eta}) S(\boldsymbol{x})\}$ of this random field, defined for all $\boldsymbol{\eta}=\left(\eta_{1}, \ldots, \eta_{d}\right)$ in $\mathbb{R}^{d}$, is written as $R_{S}(\boldsymbol{\eta})=\rho_{1}\left(\eta_{1}\right) \times \ldots \times \rho_{d}\left(\eta_{d}\right)$ in which, for all $j=1, \ldots, d$, one has $\rho_{j}(0)=1$ and $\rho_{j}\left(\eta_{j}\right)=4 L_{j}^{2} /\left(\pi^{2} \eta_{j}^{2}\right) \sin ^{2}\left(\pi \eta_{j} /\left(2 L_{j}\right)\right)$ for $\eta_{j} \neq 0$.

Properties. For all $\boldsymbol{x}$ in $\mathbb{R}^{d}, E\{S(\boldsymbol{x})\}=0$ and $E\left\{S(\boldsymbol{x})^{2}\right\}=1$. The random field $S$ is mean-square continuous on $\mathbb{R}^{d}$ ans its power spectral measure has a compact support. Introducing $L_{j}^{S}$ as the spatial correlation length relative to coordinate $x_{j}$ and defined by $L_{j}^{S}=\int_{0}^{+\infty}\left|R_{S}\left(0, \ldots, 0, \eta_{j}, 0, \ldots, 0\right)\right| d \eta_{j}$, it can be seen that $L_{j}^{S}=L_{j}$. Consequently, parameters $L_{1}, \ldots, L_{d}$ represent the spatial correlation lengths of random field $S$.

\subsection{Ensemble $S F G^{+}$}

One begins in introducing a family of non-linear functions $h$ used to define the ensemble $\mathrm{SFG}^{+}$.

Definition of the family of functions $\{u \mapsto h(\alpha, u)\}_{\alpha>0}$. Let $\alpha$ be a positive real number. The function $u \mapsto h(\alpha, u)$ from $\mathbb{R}$ into $] 0,+\infty\left[\right.$ is such that $\Gamma_{\alpha}=h(\alpha, U)$ is a gamma random variable with the parameter $\alpha$ while $U$ is a normalized Gaussian random variable $\left(E\{U\}=0\right.$ and $\left.E\left\{U^{2}\right\}=1\right)$. Consequently, for 
all $u$ in $\mathbb{R}$, we have $h(\alpha, u)=F_{\Gamma_{\alpha}}^{-1}\left(F_{U}(u)\right)$ in which $u \mapsto F_{U}(u)=P(U \leq u)$ is the cumulative distribution function of the normalized Gaussian random variable $U$. The function $p \mapsto F_{\Gamma_{\alpha}}^{-1}(p)$ from $] 0,1[$ into ] $0,+\infty\left[\right.$ is the reciprocical function of the cumulative distribution function $\gamma \mapsto F_{\Gamma_{\alpha}}(\gamma)$ from $] 0,+\infty[$ into $] 0,1\left[\right.$ of the gamma random variable $\Gamma_{\alpha}$ with the parameter $\alpha$, which is such that, for all $\gamma$ in $\mathbb{R}^{+}$, $F_{\Gamma_{\alpha}}(\gamma)=\int_{0}^{\gamma} \frac{1}{\Gamma(\alpha)} t^{\alpha-1} e^{-t} d t$ in which $\Gamma(\alpha)$ is the gamma function.

Definition of the ensemble $S F G^{+}$of the random field $\boldsymbol{x} \mapsto\left[\boldsymbol{G}_{n}(\boldsymbol{x})\right]$. The ensemble $\mathrm{SFG}^{+}$is defined as the set of all the random fields $\boldsymbol{x} \mapsto\left[\boldsymbol{G}_{n}(\boldsymbol{x})\right]$, defined on the probability space $(\Theta, \mathcal{T}, P)$, indexed by $\mathbb{R}^{d}$ where $d \geq 1$ is a fixed integer, with values in $\mathbb{M}_{n}^{+}(\mathbb{R})$ where $n \geq 2$ is another fixed integer, and defined as follows: ( i) Let $\left\{S_{j j^{\prime}}(\boldsymbol{x}), \boldsymbol{x} \in \mathbb{R}^{d}\right\}, 1 \leq j \leq j^{\prime} \leq n$, be $n(n+1) / 2$ independent copies of the random field $\left\{S(\boldsymbol{x}), \boldsymbol{x} \in \mathbb{R}^{d}\right\}$ defined in Section 2.1. Consequently, for $1 \leq j \leq j^{\prime} \leq n$, one has $E\left\{S_{j j^{\prime}}(\boldsymbol{x})\right\}=0$ and $E\left\{S_{j j^{\prime}}(\boldsymbol{x})^{2}\right\}=1$ and the random field $\boldsymbol{x} \mapsto S_{j j^{\prime}}(\boldsymbol{x})$ is completely defined. (ii) Let $\delta$ be the real number, independent of $\boldsymbol{x}$ and $n$, such that $0<\delta<\sqrt{(n+1)(n+5)^{-1}}<1$. This parameter will allow the dispersion of the random field to be controlled. ( iii) For all $\boldsymbol{x}$ in $\mathbb{R}^{d},\left[\boldsymbol{G}_{n}(\boldsymbol{x})\right]=\left[\boldsymbol{L}_{n}(\boldsymbol{x})\right]^{T}\left[\boldsymbol{L}_{n}(\boldsymbol{x})\right]$ in which $\left[\boldsymbol{L}_{n}(\boldsymbol{x})\right]$ is the upper $(n \times n)$ real triangular random matrix defined as follows. The $n(n+1) / 2$ random fields $\boldsymbol{x} \mapsto\left[\boldsymbol{L}_{n}(\boldsymbol{x})\right]_{j j^{\prime}}$ for $1 \leq j \leq j^{\prime} \leq n$, are independent. For $j<j^{\prime}$, the real-valued random field $\boldsymbol{x} \mapsto\left[\boldsymbol{L}_{n}(\boldsymbol{x})\right]_{j j^{\prime}}$, indexed by $\mathbb{R}^{d}$, is defined by $\left[\boldsymbol{L}_{n}(\boldsymbol{x})\right]_{j j^{\prime}}=\sigma_{n} S_{j j^{\prime}}(\boldsymbol{x})$ in which $\sigma_{n}$ is such that $\sigma_{n}=\delta(n+1)^{-1 / 2}$. For $j=j^{\prime}$, the positive-valued random field $\boldsymbol{x} \mapsto\left[\boldsymbol{L}_{n}(\boldsymbol{x})\right]_{j j}$, indexed by $\mathbb{R}^{d}$, is defined by $\left[\boldsymbol{L}_{n}(\boldsymbol{x})\right]_{j j}=\sigma_{n} \sqrt{2 h\left(\alpha_{j}, S_{j j}(\boldsymbol{x})\right)}$ in which, for $j=1, \ldots, n$, one hask $\alpha_{j}=(n+1) /\left(2 \delta^{2}\right)+(1-j) / 2$.

Basic properties. $\boldsymbol{x} \mapsto\left[\boldsymbol{G}_{n}(\boldsymbol{x})\right]$ is a homogeneous second-order mean-square continjuous random field indexed by $\mathbb{R}^{d}$ with values in $\mathbb{M}_{n}^{+}(\mathbb{R})$. In addition, the trajectories of random field $\boldsymbol{x} \mapsto\left[\boldsymbol{G}_{n}(\boldsymbol{x})\right]$ are continuous from $\mathbb{R}^{d}$ into $\mathbb{M}_{n}^{+}(\mathbb{R})$ almost surely. For all $\boldsymbol{x} \in \mathbb{R}^{d}$, one has $E\left\{\left\|\left[\boldsymbol{G}_{n}(\boldsymbol{x})\right]\right\|_{F}^{2}\right\}<+\infty$ and $E\left\{\left[\boldsymbol{G}_{n}(\boldsymbol{x})\right]\right\}=\left[I_{n}\right]$. The parameter $\delta$ is such that $\delta=\left\{\frac{1}{n} E\left\{\left\|\left[\boldsymbol{G}_{n}(\boldsymbol{x})\right]-\left[I_{n}\right]\right\|_{F}^{2}\right\}\right\}^{1 / 2}$ which shows that $E\left\{\left\|\left[\boldsymbol{G}_{n}(\boldsymbol{x})\right]\right\|_{F}^{2}\right\}=n\left(\delta^{2}+1\right)$. The random field $\boldsymbol{x} \mapsto\left[\boldsymbol{G}_{n}(\boldsymbol{x})\right]$ is non-Gaussian. For all $\boldsymbol{x}$ fixed in $\mathbb{R}^{d}$, the probability distribution on $\mathbb{M}_{n}^{+}(\mathbb{R})$ the random matrix $\left[\boldsymbol{G}_{n}(\boldsymbol{x})\right]$ is explicitly calculated in [2] and shows that, for all $\boldsymbol{x}$ fixed in $\mathbb{R}^{d}$, the random variables $\left\{\left[\boldsymbol{G}_{n}(\boldsymbol{x})\right]_{i j}, 1 \leq i \leq j \leq n\right\}$ are mutually dependent. The system of the marginal probability distributions of the random field $\boldsymbol{x} \mapsto\left[\boldsymbol{G}_{n}(\boldsymbol{x})\right]$ is well defined but cannot be explicitly calculated. Finally, since $\left[\boldsymbol{G}_{n}(\boldsymbol{x})\right]$ belongs to $\mathbb{M}_{n}^{+}(\mathbb{R})$ almost surely, then $\left[\boldsymbol{G}_{n}(\boldsymbol{x})\right]^{-1}$ exists almost surely. However, since almost sure convergence does not yield mean-square convergence, one does not have, a priori, $E\left\{\left\|\left[\boldsymbol{G}_{n}(\boldsymbol{x})\right]^{-1}\right\|^{2}\right\}<+\infty$. Nevertheless, it is proved in [2] that, for all $n \geq 2$ and for all $\boldsymbol{x} \in \mathbb{R}^{d}$, there exists a positive constant $c_{0}$ independent of $n$ and independent of $\boldsymbol{x}$, but depending on $\delta$, such that $E\left\{\left\|\left[\boldsymbol{G}_{n}(\boldsymbol{x})\right]^{-1}\right\|^{2}\right\} \leq c_{0}<+\infty$. 
Fundamental property. Let $\Omega$ be a bounded open domain of $\mathbb{R}^{d}$ and let $\bar{\Omega}=\Omega \cup \partial \Omega$ be its closure in which $\partial \Omega$ is the boundary of $\Omega$. One then has

$$
E\left\{\left(\sup _{\boldsymbol{x} \in \bar{\Omega}}\left\|\left[\boldsymbol{G}_{n}(\boldsymbol{x})\right]^{-1}\right\|\right)^{2}\right\}=c_{G}^{2}<+\infty
$$

in which sup is the supremum and where $0<c_{G}<+\infty$ is a finite positive constant.

Remark concerning the proof of Eq. (3). Let us consider the case $d=1$ with $\bar{\Omega}$ be a compact interval of $\mathbb{R}$. Since the stochastic process $\left\{\left\|G_{n}(\boldsymbol{x})^{-1}\right\|, \boldsymbol{x} \in \bar{\Omega} \subset \mathbb{R}\right\}$ is not a continuous local martingal with respect to an increasing family of $\sigma$-fields, the following fundamental Doob maximal inequality [3] $E\left\{\sup _{\boldsymbol{x} \in \bar{\Omega}}\left\|\left[\boldsymbol{G}_{n}(\boldsymbol{x})\right]^{-1}\right\|^{2}\right\} \leq 4 E\left\{\left\|\left[\boldsymbol{G}_{n}(\boldsymbol{x})\right]^{-1}\right\|^{2}\right\}$ cannot be used. In addition, one has to consider the non-Gaussian random field case $d \geq 2$. Consequently, there is no known result allowing a direct proof of Eq. (3) to be obtained and a complete proof of this fundamental result is given in [4].

\section{Ensemble $\mathrm{SFE}^{+}$of non-Gaussian positive-definite matrix-valued random fields}

\subsection{Definition of the ensemble $S F E^{+}$}

Let $d \geq 1$ and $n \geq 2$ be two fixed integers. Let $\Omega$ be an open (or closed) bounded (or not) domain of $\mathbb{R}^{d}$ (one can have $\Omega=\mathbb{R}^{d}$ ). Let $\boldsymbol{x} \mapsto\left[\underline{a}_{n}(\boldsymbol{x})\right]$ be a matrix-valued field from $\Omega$ into $\mathbb{M}_{n}^{+}(\mathbb{R})$. Then, for all $\boldsymbol{x}$ fixed in $\Omega$, there is an upper triangular invertible matrix $\left[\underline{L}_{n}(\boldsymbol{x})\right]$ in $\mathbb{M}_{n}(\mathbb{R})$ such that $\left[\underline{a}_{n}(\boldsymbol{x})\right]=$ $\left[\underline{L}_{n}(\boldsymbol{x})\right]^{T}\left[\underline{L}_{n}(\boldsymbol{x})\right]$. It is assumed that: ( i) there is a real positive constant $0<\underline{c}_{0}<+\infty$ independent of $\boldsymbol{x}$ such that, for all $\boldsymbol{x}$ in $\Omega$ and for all $\boldsymbol{y} \in \mathbb{R}^{n},\left\langle\left[\underline{a}_{n}(\boldsymbol{x})\right] \boldsymbol{y}, \boldsymbol{y}>\geq \underline{c}_{0}\|\boldsymbol{y}\|^{2}\right.$; (ii) there is a real positive constant $0<\underline{c}_{1}<+\infty$ independent of $\boldsymbol{x}$ such that, for all $\boldsymbol{x}$ in $\Omega$, one has $\left\|\left[\underline{L}_{n}(\boldsymbol{x})\right]\right\| \leq \sqrt{\underline{c}_{1}}$ which yields $<\left[\underline{a}_{n}(\boldsymbol{x})\right] \boldsymbol{y}, \boldsymbol{y}>\leq \underline{c}_{1}\|\boldsymbol{y}\|^{2}$, for all $\boldsymbol{y}$ in $\mathbb{R}^{n}$ and for all $\boldsymbol{x}$ in $\Omega$. Consequently, for all $\boldsymbol{x}$ in $\Omega$, one has $\left\|\left[\underline{a}_{n}(\boldsymbol{x})\right]\right\| \leq \underline{c}_{1}$ and $\left\|\left[\underline{a}_{n}(\boldsymbol{x})\right]\right\|_{F} \leq \sqrt{n} \underline{c}_{1}$. The ensemble $\mathrm{SFE}^{+}$is then defined as the set of all the random fields $\boldsymbol{x} \mapsto\left[\boldsymbol{A}_{n}(\boldsymbol{x})\right]$, defined on the probability space $(\Theta, \mathcal{T}, P)$, indexed by $\Omega$, with values in $\mathbb{M}_{n}^{+}(\mathbb{R})$, such that

$$
\forall \boldsymbol{x} \in \Omega \quad, \quad\left[\boldsymbol{A}_{n}(\boldsymbol{x})\right]=\left[\underline{L}_{n}(\boldsymbol{x})\right]^{T}\left[\boldsymbol{G}_{n}(\mathbf{x})\right]\left[\underline{L}_{n}(\boldsymbol{x})\right],
$$

in which $\boldsymbol{x} \mapsto\left[\boldsymbol{G}_{n}(\boldsymbol{x})\right]$ is the random field in $\mathrm{SFG}^{+}$, defined on $(\Theta, \mathcal{T}, P)$, indexed by $\mathbb{R}^{d}$ and with values in $\mathbb{M}_{n}^{+}(\mathbb{R})$ (see Section 2.2). 


\subsection{Properties of the random field $\boldsymbol{x} \mapsto\left[\boldsymbol{A}_{n}(\boldsymbol{x})\right]$}

Basic properties. For all $\boldsymbol{x}$ in $\Omega,\left[\boldsymbol{A}_{n}(\boldsymbol{x})\right]$ is a random matrix with values in $\mathbb{M}_{n}^{+}(\mathbb{R})$, the mean function is $\boldsymbol{x} \mapsto E\left\{\left[\boldsymbol{A}_{n}(\boldsymbol{x})\right]\right\}=\left[\underline{a}_{n}(\boldsymbol{x})\right] \in \mathbb{M}_{n}^{+}(\mathbb{R})$ and $E\left\{\left\|\left[\boldsymbol{A}_{n}(\boldsymbol{x})\right]\right\|^{2}\right\} \leq E\left\{\left\|\left[\boldsymbol{A}_{n}(\boldsymbol{x})\right]\right\|_{F}^{2}\right\}<+\infty$ which proves that $\boldsymbol{x} \mapsto\left[\boldsymbol{A}_{n}(\boldsymbol{x})\right]$ is a second-order random field on $\Omega$. In general, since $\left[\underline{a}_{n}(\boldsymbol{x})\right]$ depends on $\boldsymbol{x}$, then the random field $\left\{\left[\boldsymbol{A}_{n}(\boldsymbol{x})\right], \boldsymbol{x} \in \Omega\right\}$ is non homogeneous. We have $E\left\{\left\|\left[\boldsymbol{A}_{n}(\boldsymbol{x})\right]-\left[\underline{a}_{n}(\boldsymbol{x})\right]\right\|_{F}^{2}\right\}=\left\{\delta^{2} /(n+1)\right\}\left\{\left\|\left[\underline{a}_{n}(\boldsymbol{x})\right]\right\|_{F}^{2}+\right.$ $\left.\left(\operatorname{tr}\left[\underline{a}_{n}(\boldsymbol{x})\right]\right)^{2}\right\}$. The dispersion parameter, defined by $\delta_{A_{n}}(\boldsymbol{x})=\left\{E\left\{\left\|\left[\boldsymbol{A}_{n}(\boldsymbol{x})\right]-\left[\underline{a}_{n}(\boldsymbol{x})\right]\right\|_{F}^{2}\right\} /\left\|\left[\underline{a}_{n}(\boldsymbol{x})\right]\right\|_{F}^{2}\right\}^{1 / 2}$, is such that $\delta_{A_{n}}(\boldsymbol{x})=(\delta / \sqrt{n+1})\left\{1+\left(\operatorname{tr}\left[\underline{a}_{n}(\boldsymbol{x})\right]\right)^{2} / \operatorname{tr}\left\{\left[\underline{a}_{n}(\boldsymbol{x})\right]^{2}\right\}\right\}^{1 / 2}$.

Spatial correlation lengths for the homogeneous case. If $\left[\underline{a}_{n}(\boldsymbol{x})\right]=\left[\underline{a}_{n}\right]$ is independent of $\boldsymbol{x}$, then the ran$\operatorname{dom}$ field $\left\{\left[\boldsymbol{A}_{n}(\boldsymbol{x})\right]=\left[\underline{L}_{n}\right]^{T}\left[\boldsymbol{G}_{n}(\boldsymbol{x})\right]\left[\underline{L}_{n}\right], \boldsymbol{x} \in \Omega\right\}$ can be viewed as the restriction to $\Omega$ of a homogeneous random field indexed by $\mathbb{R}^{d}$. Therefore, $\delta_{A_{n}}(\boldsymbol{x})=\delta_{A_{n}}$ is independent of $\boldsymbol{x}$. Let $\boldsymbol{\eta}=\left(\eta_{1}, \ldots, \eta_{d}\right) \mapsto$ $r^{A_{n}}(\boldsymbol{\eta})=\operatorname{tr} E\left\{\left(\left[\boldsymbol{A}_{n}(\boldsymbol{x}+\boldsymbol{\eta})\right]-\left[\underline{a}_{n}\right]\right)\left(\left[\boldsymbol{A}_{n}(\boldsymbol{x})\right]-\left[\underline{a}_{n}\right]\right)\right\} / E\left\{\left\|\left[\boldsymbol{A}_{n}(\boldsymbol{x})\right]-\left[\underline{a}_{n}\right]\right\|_{F}^{2}\right\}$ from $\mathbb{R}^{d}$ into $\mathbb{R}$. One has $r^{A_{n}}(0)=1$ and $r^{A_{n}}(-\boldsymbol{\eta})=r^{A_{n}}(\boldsymbol{\eta})$. For all $j=1, \ldots, d$, the spatial correlation length $L_{j}^{A_{n}}$ of the homogeneous random field $\boldsymbol{x} \mapsto\left[\boldsymbol{A}_{n}(\boldsymbol{x})\right]$ indexed by $\mathbb{R}^{d}$, relative to the coordinate $x_{j}$, can then be defined by $L_{j}^{A_{n}}=\int_{0}^{+\infty}\left|r^{A_{n}}\left(0, \ldots, 0, \eta_{j}, 0, \ldots, 0\right)\right| d \eta_{j}$.

\section{Ellipticity of the stochastic elasticity operator}

The stochastic elasticity operator given by Eq. (2) is assumed to be defined on an open bounded domain $\Omega$ of $\mathbb{R}^{3}$ whose boundary $\partial \Omega$ is written as $\Gamma_{0} \cup \Gamma$. On $\Gamma_{0}$, there is a zero Dirichlet boundary condition. One introduces the real Hilbert spaces $H=\left(L^{2}(\Omega)\right)^{3}$ and $V=\left\{\boldsymbol{u} \in\left(H^{1}(\Omega)\right)^{3}, \boldsymbol{u}=0\right.$ on $\left.\Gamma_{0}\right\}$ whose inner products are denoted by $\langle\boldsymbol{u}, \boldsymbol{w}\rangle_{H}$ and $\langle\boldsymbol{u}, \boldsymbol{w}\rangle_{V}$ respectively, and where the associated norms are denoted by $\|\boldsymbol{u}\|_{H}$ and $\|\boldsymbol{u}\|_{V}$ respectively. Let $\mathbb{H}=L^{2}(\Theta, H)$ and $\left.\mathbb{V}=L^{2}(\Theta, V)\right)$ be the real Hilbert spaces of all the second-order random variables $\theta \mapsto\{\boldsymbol{x} \mapsto \boldsymbol{U}(\boldsymbol{x}, \theta)\}$ defined on the probability space $(\Theta, \mathcal{T}, P)$, with values in $H$ and $V$ respectively, equipped with the inner products $\ll \boldsymbol{U}, \boldsymbol{W} \gg_{\mathbb{H}}=E\left\{\langle\boldsymbol{U}, \boldsymbol{W}\rangle_{H}\right\}$ and $\left.\ll \boldsymbol{U}, \boldsymbol{W} \gg_{\mathbb{V}}=E\{<\boldsymbol{U}, \boldsymbol{W}\rangle_{V}\right\}$ respectively, and where the associated norms are denoted by $\|\boldsymbol{U}\|_{\mathbb{H}}$ and $\|\boldsymbol{U}\|_{\mathbb{V}}$ respectively.

\subsection{Weak formulation of the stochastic elasticity operator}

Let $n=6$ and $d=3$. Let us introduce the new indices $I$ and $J$ belonging to $\{1, \ldots, 6\}$ such that $I=(i, j)$ and $J=(k, h)$ with the following correspondence: $1=(1,1), 2=(2,2), 3=(3,3), 4=(1,2), 5=(2,3)$ and $6=(3,1)$. Thus, for all $\boldsymbol{x}$ in $\Omega$, one introduces the matrix $\left[\underline{a}_{n}(\boldsymbol{x})\right]$ in $\mathbb{M}_{n}^{+}(\mathbb{R})$ such that $\left[\underline{a}_{n}(\boldsymbol{x})\right]_{I J}=$ 
$\underline{c}_{i j k h}(\boldsymbol{x})$ and the random $(n \times n)$ real matrix $\left[\boldsymbol{A}_{n}(\boldsymbol{x})\right]$ such that $\left[\boldsymbol{A}_{n}(\boldsymbol{x})\right]_{I J}=C_{i j k h}(\boldsymbol{x})$. A nonparametric probabilistic model of the random fourth-order elasticity tensor $C_{i j k h}(\boldsymbol{x})$ consists in choosing the random field $\boldsymbol{x} \mapsto\left[\boldsymbol{A}_{n}(\boldsymbol{x})\right]$ in $\mathrm{SFE}^{+}$with the mean value $\left[\underline{a}_{n}(\boldsymbol{x})\right]=E\left\{\left[\boldsymbol{A}_{n}(\boldsymbol{x})\right]\right\}$. The weak formulation of the stochastic elasticity operator defined by Eq. (2) leads the random bilinear form $(\boldsymbol{U}, \boldsymbol{W}) \mapsto \boldsymbol{K}(\boldsymbol{U}, \boldsymbol{W})$ on $\mathbb{V} \times \mathbb{V}$ to be introduced, such that

$$
\boldsymbol{K}(\boldsymbol{U}, \boldsymbol{W})=\int_{\Omega}<\left[\boldsymbol{A}_{n}(\boldsymbol{x})\right] \boldsymbol{e}(\boldsymbol{U}(\boldsymbol{x})), \boldsymbol{e}(\boldsymbol{W}(\boldsymbol{x}))>d \boldsymbol{x}
$$

in which $\boldsymbol{e}(\boldsymbol{u})=\left(\varepsilon_{11}(\boldsymbol{u}), \varepsilon_{22}(\boldsymbol{u}), \varepsilon_{33}(\boldsymbol{u}), 2 \varepsilon_{12}(\boldsymbol{u}), 2 \varepsilon_{23}(\boldsymbol{u}), 2 \varepsilon_{31}(\boldsymbol{u})\right)$.

\subsection{Ellipticity of the random bilinear form}

Let $(\boldsymbol{U}, \boldsymbol{W}) \mapsto \mathcal{K}(\boldsymbol{U}, \boldsymbol{W})$ be the bilinear form on $\mathbb{V} \times \mathbb{V}$ defined by $\mathcal{K}(\boldsymbol{U}, \boldsymbol{W})=E\{\boldsymbol{K}(\boldsymbol{U}, \boldsymbol{W})\}$. If the following property was introduced: for all $\boldsymbol{x} \in \Omega$ and for all $\mathbb{R}^{n}$-valued random variable $\boldsymbol{Y}$ defined on $(\Theta, \mathcal{T}, P),<\left[\boldsymbol{A}_{n}(\boldsymbol{x})\right] \boldsymbol{Y}, \boldsymbol{Y}>\geq c\|\boldsymbol{Y}\|^{2}$ a.s in which $0<c<+\infty$ is independent of $\boldsymbol{x}$, then the bilinear form $(\boldsymbol{U}, \boldsymbol{W}) \mapsto \mathcal{K}(\boldsymbol{U}, \boldsymbol{W})$ on $\mathbb{V} \times \mathbb{V}$ would be coercive in $\mathbb{V}$ (i.e. $\mathbb{V}$-elliptic) because, we would have $\mathcal{K}(\boldsymbol{U}, \boldsymbol{U}) \geq c E\left\{\int_{\Omega}\|\boldsymbol{e}(\boldsymbol{U}(\boldsymbol{x}))\|^{2} d \boldsymbol{x}\right\} \geq c_{\mathcal{K}}\|\boldsymbol{U}\|_{\mathbb{V}}^{2}$ with $0<c_{\mathcal{K}}<+\infty$. This uniform ellipticity condition, which is generally not coherent with the available information which can be deduced from the objective data, does not hold for the random field $\boldsymbol{x} \mapsto\left[\boldsymbol{A}_{n}(\boldsymbol{x})\right]$ belonging to $\mathrm{SFE}^{+}$and consequently, the usual analysis given above cannot presently be used. A non uniform ellipticity condition has to be developed using the fundamental property defined by Eq. (3): it is proved [4] that, for all random field $\{\boldsymbol{x} \mapsto \boldsymbol{U}(\boldsymbol{x})\}$ in $\mathbb{V}$, we have

$$
\sqrt{E\left\{\boldsymbol{K}(\boldsymbol{U}, \boldsymbol{U})^{2}\right\}} \geq c_{K}\|\boldsymbol{U}\|_{\mathbb{V}}^{2}
$$

in which $c_{K}$ is a positive finite real constant. Note that Eq. (6) differs from $E\{\boldsymbol{K}(\boldsymbol{U}, \boldsymbol{U})\} \geq c_{\mathcal{K}}\|\boldsymbol{U}\|_{\mathbb{V}}^{2}$ due to the fact that the two positive-valued random variables $\sup _{\boldsymbol{x} \in \bar{\Omega}}\left\|\left[\boldsymbol{G}_{n}(\boldsymbol{x})\right]^{-1}\right\|$ and $\boldsymbol{K}(\boldsymbol{U}, \boldsymbol{U})$ are dependent.

\subsection{Existence and uniqueness of a weak second-order stochastic solution for a stochastic BVP}

Let $\boldsymbol{w} \mapsto f(\boldsymbol{w})$ be a given continuous linear form on $V$, that is to say such that $|f(\boldsymbol{w})| \leq c_{f}\|\boldsymbol{w}\|_{V}$ with $0<c_{f}<+\infty$. Then, the following random problem: find a random field $\{\boldsymbol{x} \mapsto \boldsymbol{U}(\boldsymbol{x})\}$ in $\mathbb{V}$ such that, for all $\boldsymbol{W} \in \mathbb{V}, \boldsymbol{K}(\boldsymbol{U}, \boldsymbol{W})=f(\boldsymbol{W})$ a.s, has a unique stochastic solution $\{\boldsymbol{x} \mapsto \boldsymbol{U}(\boldsymbol{x})\}$ in $\mathbb{V}$.

The proof can easily be constructed. From equations $\boldsymbol{K}(\boldsymbol{U}, \boldsymbol{W})=f(\boldsymbol{W})$ and $|f(\boldsymbol{w})| \leq c_{f}\|\boldsymbol{w}\|_{V}$, it can be deduced that $\boldsymbol{K}(\boldsymbol{U}, \boldsymbol{U}) \leq c_{f}\|\boldsymbol{U}\|_{V}$ and consequently, $E\left\{\boldsymbol{K}(\boldsymbol{U}, \boldsymbol{U})^{2}\right\} \leq c_{f}^{2} E\left\{\|\boldsymbol{U}\|_{V}^{2}\right\}$. Using Eq. (6) 
yields $c_{K}^{2}\|\boldsymbol{U}\|_{\mathbb{V}}^{4} \leq c_{f}^{2}\|\boldsymbol{U}\|_{\mathbb{V}}^{2}$ which can be rewritten as $\|\boldsymbol{U}\|_{\mathbb{V}} \leq c_{U}<+\infty$ with $c_{U}=c_{f} / c_{K}$ and yields the existence. Finally, the proof of the uniqueness is straightforward because, if $\boldsymbol{U}$ and $\boldsymbol{U}^{\prime}$ are two solutions in $\mathbb{V}$, for all $\boldsymbol{W}$ in $\mathbb{V}$, one has $\boldsymbol{K}\left(\boldsymbol{U}-\boldsymbol{U}^{\prime}, \boldsymbol{W}\right)=0$ a.s and thus $E\left\{\boldsymbol{K}\left(\boldsymbol{U}-\boldsymbol{U}^{\prime}, \boldsymbol{W}\right)^{2}\right\}=0$. Taking $\boldsymbol{W}=\boldsymbol{U}-\boldsymbol{U}^{\prime}$ and from Eq. (6) yield $\left\|\boldsymbol{U}-\boldsymbol{U}^{\prime}\right\|_{\mathbb{V}}^{2}=0$, i.e., $\boldsymbol{U}=\boldsymbol{U}^{\prime}$ in $\mathbb{V}$.

\section{Conclusions}

One has presented the mathematical construction of a non-Gaussian positive-definite $(n \times n)$ real matrixvalued random field, indexed by any domain of $\mathbb{R}^{d}$, depending only on the mean function and on a small number of scalar parameters constituted of a dispersion parameter and $d$ spatial correlation lengths. Such a random field is adapted to the stochastic inverse problem relative to the experimental identification of the random field. A fundamental mathematical property is proved and allows the ellipticity of stochastic partial differential operators to be obtained.

\section{References}

[1] P. G. Ciarlet, Mathematical Elasticity, Volume 1: Three Dimensional Elasticity, North-Holland, Amsterdam, 1988.

[2] C. Soize, Maximum entropy approach for modeling random uncertainties in transient elastodynamics, Journal of the Acoustical Society of Amererica 109(5) (2001) 1979-1996.

[3] J.L. Doob, Stochastic Processes, John Wiley and Sons, New York, 1953 (Wiley Classics Library Edition Published 1990).

[4] C. Soize, Non Gaussian positive-definite matrix-valued random fields for elliptic stochastic partial differential operators, Computer Methods in Applied Mechanics and Engineering, submitted in March 2004. 